\title{
Frequency shift of hyperfine transitions due to blackbody radiation
}

\author{
E. J. Angstmann, V. A. Dzuba, and V. V. Flambaum \\ School of Physics, University of New South Wales, Sydney 2052, Australia
}

(Dated: October 15, 2018)

\begin{abstract}
We have performed calculations of the size of the frequency shift induced by a static electric field on the clock transition frequencies of the hyperfine splitting in $\mathrm{Yb}^{+}, \mathrm{Rb}, \mathrm{Cs}, \mathrm{Ba}^{+}, \mathrm{and}_{\mathrm{Hg}}^{+}$. The calculations are used to find the frequency shifts due to blackbody radiation which are needed for accurate frequency measurements and improvements of the limits on variation of the fine structure constant, $\alpha$. Our result for Cs $\left(\delta \nu / E^{2}=-2.26(2) \times 10^{-10} \mathrm{~Hz} /(\mathrm{V} / \mathrm{m})^{2}\right)$ is in good agreement with early measurements and $a b$ initio calculations. We present arguments against recent claims that the actual value might be smaller. The difference $(\sim 10 \%)$ is due to the contribution of the continuum spectrum in the sum over intermediate states.
\end{abstract}

PACS numbers: 32.60.+i,31.30.Gs,31.25.Eb

\section{INTRODUCTION}

Atomic clocks are now important for both practical applications and fundamental physics. The hyperfine structure (hfs) transition of the ground state of ${ }^{133}$ Cs serves as a primary frequency standard providing the definition of a metric second. Many similar hfs transitions in other atoms and ions are used or under consideration for use as secondary microwave frequency standards. Most frequency standards (atomic clocks) operate at room temperature. However, the exact definition of a metric second corresponds to the frequency of the transition measured at zero temperature. This means that readings from atomic clocks should be corrected to account for the effect of black body radiation (see e.g. [1]). The value of this effect can be found from measurements or calculations. There are many experimental 2, 3, 4, 5, 6, 7] and theoretical 1, 8, 9, 10, 11, 12, 13] works studying the effects of black body radiation on microwave frequency standards. However, the situation is far from being satisfactory. There is disagreement among the different works for cesium which will be discussed in more detail below. On the other hand, the data for other atoms and ions is very poor or absent.

In the present work we perform accurate calculations of the shift due to blackbody radiation in the hyperfine transitions in $\mathrm{Yb}^{+}, \mathrm{Rb}, \mathrm{Cs}, \mathrm{Ba}^{+}$, and $\mathrm{Hg}^{+}$. These transitions play key roles in microwave frequency standards. They are also of interest to physicists considering experiments to measure $\alpha$ variation in the laboratory (see e.g. 14, 15, 16, 17]). An experiment is currently planned utilizing $\mathrm{Yb}^{+}[18]$. No accurate calculations or measurements of the radiation shift for this ion have been performed previously. However, calculations or measurements are available for other atoms and ions 1, 2, 3, 4, 5, 6, 7, 8, 9, 10, 11, 12, 13]. We compare our results with these values.

Due to the importance of cesium as a primary frequency standard we have performed a more detailed study of this atom. This was the subject of a separate short paper [19]. In the present paper we give more details of the calculations while also discussing other atoms and ions.

There is some disagreement on the actual value of the radiation frequency shift for cesium. Early measurements [2, 3, 5] and ab initio calculations [9, 10] support a value which is close to $-2.2 \times 10^{-10} \mathrm{~Hz} /(\mathrm{V} / \mathrm{m})^{2}$ while more recent measurements [6, 7] and semiempirical calculations 8, 11, 12] claim that the actual number might be about $10 \%$ smaller. While we cannot comment on the experimental results, the source of disagreement between theoretical values seems to be in the continuum spectrum. Ab initio calculations (including the present work) include the contribution of the continuum spectrum into the summation over the complete set of states while semiempirical calculations do not. We demonstrate that adding the contribution of the continuum spectrum to where it was missed brings all theoretical results into good agreement with each other and with early measurements.

\section{THEORY}

Blackbody radiation creates a temperature dependent electric field, described by the Planck radiation law

$$
E^{2}(\omega)=\frac{8 \alpha}{\pi} \frac{\omega^{3} d \omega}{\exp \left(\omega / k_{B} T\right)-1} .
$$

This leads to the following expression for the average electric field radiated by a black body at temperature T:

$$
\left\langle E^{2}\right\rangle=(831.9 \mathrm{~V} / \mathrm{m})^{2}[\mathrm{~T}(\mathrm{~K}) / 300]^{4} .
$$

This electric field causes a temperature-dependent frequency shift of the atomic microwave clock transitions. It can be presented in the form (see, e.g. 1] )

$$
\delta \nu / \nu_{0}=\beta\left(T / T_{0}\right)^{4}\left[1+\epsilon\left(T / T_{0}\right)^{2}\right] .
$$

Here $T_{0}$ is usually assumed to be room temperature $\left(T_{0}=\right.$ $300 K)$. The frequency shift in a static electric field is

$$
\delta \nu=k E^{2} .
$$


The coefficients $k$ and $\beta$ are related by

$$
\beta=\frac{k}{\nu_{0}}(831.9 \mathrm{~V} / \mathrm{m})^{2},
$$

$\epsilon$ is a small correction due to frequency distribution (11). In the present work we consider both terms in (3) by calculating coefficients $k, \beta$ and $\epsilon$.

It is convenient to start from calculation of $k$ by considering an atom in static electric field. In the case when there is no other external electric field which sets preferred direction the radiation shift can be expressed in terms of the scalar hyperfine polarizability of the atom. This corresponds to averaging over all possible directions of the electric field. The hyperfine polarizability is the difference of the atomic polarizabilities between different hyperfine structure states of the atom. The lowest-order effect is linear in the hyperfine interaction and quadratic in the electric field. Therefore, its value can be calculated using third-order perturbation theory (see, e.g. 26])

$$
\begin{aligned}
\delta \epsilon_{a} & =\sum_{n, m} \frac{\langle a|\hat{V}| n\rangle\langle n|\hat{V}| m\rangle\langle m|\hat{V}| a\rangle}{\left(\epsilon_{a}-\epsilon_{n}\right)\left(\epsilon_{a}-\epsilon_{m}\right)} \\
& -\langle a|\hat{V}| a\rangle \sum_{n} \frac{\langle a|\hat{V}| n\rangle^{2}}{\left(\epsilon_{a}-\epsilon_{n}\right)^{2}} .
\end{aligned}
$$

In our case the perturbation operator $\hat{V}$ is the sum of the hyperfine structure operator and the electric dipole operator

$$
\hat{V}=\hat{H}_{h f s}-e \boldsymbol{E} \cdot \boldsymbol{r} .
$$

The operator of the hyperfine interaction $\hat{H}_{h f s}$ is given by

$$
\hat{H}_{h f s}=\frac{|e|}{c} \boldsymbol{\mu} \cdot \frac{\boldsymbol{r} \times \boldsymbol{\alpha}}{r_{>}^{3}}, \quad r_{>}=\max \left(r, r_{N}\right),
$$

where $\boldsymbol{\alpha}$ is the Dirac matrix, $\boldsymbol{\mu}$ is the magnetic moment of the nucleus and $r_{N}$ is nuclear radius.

To get the effect of the electric field on the frequency of the hyperfine transition one needs to go through the following steps:

- Substitute the perturbation operator $\hat{V}$ into equation (6).

- Keep only terms linear in $\hat{H}_{h f s}$ and quadratic in the electric field.

- Apply equation (6) to both components of the hyperfine structure doublet.

- Take the difference.

The resulting expression for the frequency shift consists of three terms. The first two of them originate from the first term of equation (6). In one of them the $\hat{H}_{h f s}$ operator is either on the left or right side of the expression (these two terms are equal and can be combined together), and in the other the $\hat{H}_{h f s}$ operator is in the middle. The last term is due to change of the normalization of the wave function (second term of equation (6)). It is proportional to the hyperfine structure of the ground state.

After angular reduction these three terms become

$$
\begin{gathered}
\delta \nu_{1}(a s)=e^{2}\left\langle E^{2}\right\rangle \frac{2 I+1}{6} \times \\
\sum_{n, m, j} \frac{A_{a s, n s}\left\langle n s\|r\| m p_{j}\right\rangle\left\langle m p_{j}\|r\| a s\right\rangle}{\left(\epsilon_{a s}-\epsilon_{n s}\right)\left(\epsilon_{a s}-\epsilon_{m p_{j}}\right)}, \\
\delta \nu_{2}(a s)=\frac{e^{2}\left\langle E^{2}\right\rangle}{6} \sum_{j}\left(C_{I+1 / 2}-C_{I-1 / 2}\right) \times \\
\sum_{n, m} \frac{\langle a s\|r\| n p j\rangle A_{n p j, m p j}\left\langle m p_{j}\|r\| a s\right\rangle}{\left(\epsilon_{a s}-\epsilon_{n p j}\right)\left(\epsilon_{a s}-\epsilon_{m p_{j}}\right)},
\end{gathered}
$$

and

$$
\begin{aligned}
& \delta \nu_{n o r m}(a s)= \\
& -e^{2}\left\langle E^{2}\right\rangle \frac{2 I+1}{12} A_{a s} \sum_{m, j} \frac{\left|\left\langle a s\|r\| m p_{j}\right\rangle\right|^{2}}{\left(\epsilon_{a s}-\epsilon_{m p_{j}}\right)^{2}} .
\end{aligned}
$$

Here

$$
\begin{aligned}
& C_{F}=\sum_{F^{\prime}}\left(2 F^{\prime}+1\right)\left[F^{\prime}\left(F^{\prime}+1\right)-I(I+1)-j(j+1)\right] \\
& \times\left\{\begin{array}{rrr}
1 / 2 & F & I \\
F^{\prime} & j & 1
\end{array}\right\}^{2}, \quad F^{\prime}=|I-J|, I+J,
\end{aligned}
$$

$A_{n s}$ is the hfs constant of the $n s$ state, $A_{m, n}$ is the offdiagonal hfs matrix element, $I$ is nuclear spin, $\mathbf{F}=\mathbf{I}+\mathbf{J}$, $\mathbf{J}$ is total electron momentum of the atom in the ground state $(J=1 / 2$ for atoms considered in present work $)$, and $j$ is total momentum of virtual $p$-states $(j=1 / 2,3 / 2)$. Summation goes over a complete set of $n s, m p_{1 / 2}$ and $m p_{3 / 2}$ states.

Expression (8) does not include the $s-d$ hfs matrix elements while expression (9) does not include the $p_{1 / 2}-p_{3 / 2}$ hfs matrix elements. Test calculations show that the total contribution of the off-diagonal (in total momentum $j$ ) hfs matrix elements is of the order of $0.1 \%$ of the final answer and therefore can be neglected in present calculations.

Expressions (8), (9) and (10) correspond to static limit when energy of thermal photon is zero. To take into account distribution (11) one needs to make the following substitutions in terms (8) and (9):

$$
\frac{\left\langle m p_{j}\|r\| \mid a s\right\rangle}{\Delta \epsilon_{s p}} \rightarrow \frac{1}{2}\left[\frac{\left\langle m p_{j}\|r\| a s\right\rangle}{\Delta \epsilon_{s p}+\omega}+\frac{\left\langle m p_{j}\|r\| a s\right\rangle}{\Delta \epsilon_{s p}-\omega}\right],
$$

and in term (10)

$$
\frac{1}{\Delta \epsilon_{s p}^{2}} \rightarrow \frac{1}{2}\left[\frac{1}{\left(\Delta \epsilon_{s p}+\omega\right)^{2}}+\frac{1}{\left(\Delta \epsilon_{s p}-\omega\right)^{2}}\right]
$$


where $\omega$ is the frequency of thermal photon. Integrating resulting expression with (1) and keeping only terms up to $\omega^{2}$ (since $\omega \ll \Delta \epsilon_{s p}$ ) leads to expression of the form (3) in which first term is given by (8), (9) and (10) and parameter $\epsilon$ in second term is given by

$$
\epsilon=\frac{A}{k}\left[\sum_{i} \frac{k_{i}^{(1)}}{\Delta \epsilon_{s p i}^{2}}+\sum_{i} \frac{k_{i}^{(2)}}{\Delta \epsilon_{s p i}^{2}}+3 \sum_{i} \frac{k_{i}^{(3)}}{\Delta \epsilon_{s p i}^{2}}\right] .
$$

Here index $i$ replaces all indexes of summation in (8), (9) and (10), $k_{i}^{(1)}$ corresponds to terms in (8), $k_{i}^{(2)}$ corresponds to (91), $k_{i}^{(3)}$ corresponds to (10) and $k=$ $k^{(1)}+k^{(2)}+k^{(3)} . \Delta \epsilon_{s p i}$ is the energy of the $s-p$ transition number $i$. If energies $\Delta \epsilon_{s p i}$ are in atomic units then $A=1.697 \times 10^{-5}$ (atomic unit of energy is $27.211 \mathrm{eV}=$ $315773 \mathrm{~K}$ ). Lowest $s-p$ transitions (e.g., $6 s-6 p_{1 / 2}$ and $6 s-6 p_{3 / 2}$ ) strongly dominate in the summation (13).

\section{CALCULATIONS}

In order to calculate frequency shift to the hfs transitions due to the electric field one needs to have a complete set of states and to have the energies, electric dipole transition amplitudes and hyperfine structure matrix elements corresponding to these states. It is possible to consider summation over the physical states and use experimental data to perform the calculations. The lowest valence states for which experimental data is usually available dominate in the summation. Off-diagonal hfs matrix elements can be obtained to a high accuracy as the square root of the product of corresponding hfs constants: $A_{m, n}=\sqrt{A_{m} A_{n}}$ (see, e.g. [27]). However, the accuracy of this approach is limited by the need to include the tail contribution from highly excited states including states in the continuum. This contribution can be very significant and its calculation is not easier than the calculation of the whole sum. Also, for atoms like $\mathrm{Yb}^{+}$and $\mathrm{Hg}^{+}$available experimental data is insufficient to follow this path.

Therefore, in the present work we use an ab initio approach in which high accuracy is achieved by the inclusion of all important many-body and relativistic effects. We make only one exception toward the semiempirical approach. The frequency shift is dominated by the term (10) which is proportional to the hfs in the ground state. These hfs constants are known to very high accuracy from measurements for all atoms considered in the present work. It is natural to use experimental hfs constants in the dominating term to have more accurate results. Note however that the difference with complete $a b$ initio calculations is small. It is also instructive to perform calculations of the hfs and atomic polarizabilities to demonstrate the accuracy of the method.

Calculations start from the relativistic Hartree-Fock (RHF) method in the $V^{N-1}$ approximation. This means that the initial RHF procedure is done for a closed-shell atomic core with the valence electron removed. After that, states of the external electron are calculated in the field of the frozen core. Correlations are included by means of the correlation potential method [28]. We use two different approximations for the correlation potential, $\hat{\Sigma}$. First, we calculate it in the lowest, second-order of the many-body perturbation theory (MBPT). We use notation $\hat{\Sigma}^{(2)}$ for the corresponding correlation potential. Then we also include into $\hat{\Sigma}$ two classes of the higherorder terms: screening of the Coulomb interaction and hole-particle interaction (see, e.g. 29] for details). These two effects are included in all orders of the MBPT and the corresponding correlation potential is named $\hat{\Sigma}^{(\infty)}$.

To calculate $\hat{\Sigma}^{(2)}$ we need a complete set of singleelectron orbitals. We use the B-spline technique [30, 31] to construct the basis. The orbitals are built as linear combinations of $50 \mathrm{~B}$-splines in a cavity of radius $40 a_{B}$. The coefficients are chosen from the condition that the orbitals are eigenstates of the RHF Hamiltonian $\hat{H}_{0}$ of the closed-shell core. The $\hat{\Sigma}^{(\infty)}$ operator is calculated with the technique which combines solving equations for the Green functions (for the direct diagram) with the summation over complete set of states (exchange diagram) 29].

The correlation potential $\hat{\Sigma}$ is then used to build a new set of single-electron states, the so-called Brueckner orbitals. This set is to be used in the summation in equations (8), (91) and (10). Here again we use the B-spline technique to build the basis. The procedure is very similar to the construction of the RHF B-spline basis. The only difference is that new orbitals are now the eigenstates of the $\hat{H}_{0}+\hat{\Sigma}$ Hamiltonian, where $\hat{\Sigma}$ is either $\hat{\Sigma}^{(2)}$ or $\hat{\Sigma}^{(\infty)}$.

We use the all-order correlation potential $\hat{\Sigma}^{(\infty)}$ for Rb, $\mathrm{Cs}$ and $\mathrm{Ba}^{+}$. It has been demonstrated in a number of works (see, e.g. 29, 32, 33]) that inclusion of the screening of Coulomb interaction and the hole-particle interaction leads to very accurate results for alkali atoms. However, for other atoms with one external electron above closed shells these two higher-order effects are not dominating and their inclusion generally does not improve the results. Therefore, for the $\mathrm{Yb}^{+}$and $\mathrm{Hg}^{+}$ions we use only the second-order correlation potential $\hat{\Sigma}^{(2)}$.

Brueckner orbitals which correspond to the lowest valence states are good approximations to the real physical states. Their quality can be checked by comparing experimental and theoretical energies. Moreover, their quality can be further improved by rescaling the correlation potential $\hat{\Sigma}$ to fit experimental energies exactly. We do this by replacing the $\hat{H}_{0}+\hat{\Sigma}$ with the $\hat{H}_{0}+\lambda \hat{\Sigma}$ Hamiltonian in which the rescaling parameter $\lambda$ is chosen for each partial wave to fit the energy of the first valence state. The values of $\lambda$ are presented in Table Note that all values are very close to unity. This means that even without rescaling the accuracy is very good and only a small adjustment to the value of $\hat{\Sigma}$ is needed. Note also that since the rescaling procedure affects both energies and wave functions, it usually leads to improved values of the matrix elements of external fields. In fact, this is 
TABLE I: Rescaling parameters for the $\hat{\Sigma}$ operator which fit energies of the lowest $s$ and $p$ states of $\mathrm{Rb}, \mathrm{Cs}, \mathrm{Ba},{ }^{+}, \mathrm{Yb}^{+}$ and $\mathrm{Hg}^{+}$.

\begin{tabular}{lllll}
\hline \hline Atom & $\hat{\Sigma}$ & $s_{1 / 2}$ & $p_{1 / 2}$ & $p_{3 / 2}$ \\
\hline $\mathrm{Rb}$ & $\hat{\Sigma}^{(2)}$ & 0.868 & 0.903 & 0.906 \\
$\mathrm{Rb}$ & $\hat{\Sigma}^{(\infty)}$ & 1.008 & 0.974 & 0.976 \\
$\mathrm{Cs}$ & $\hat{\Sigma}^{(2)}$ & 0.802 & 0.848 & 0.852 \\
$\mathrm{Cs}$ & $\hat{\Sigma}^{(\infty)}$ & 0.985 & 0.95 & 0.95 \\
$\mathrm{Ba}^{+}$ & $\hat{\Sigma}^{(2)}$ & 0.782 & 0.830 & 0.833 \\
$\mathrm{Ba}^{+}$ & $\hat{\Sigma}^{(\infty)}$ & 0.988 & 0.963 & 0.967 \\
$\mathrm{Yb}^{+}$ & $\hat{\Sigma}^{(2)}$ & 0.866 & 1.09 & 1.185 \\
$\mathrm{Hg}^{+}$ & $\hat{\Sigma}^{(2)}$ & 0.805 & 0.890 & 0.926 \\
\hline \hline
\end{tabular}

a semiempirical method to include omitted higher-order correlation corrections.

Matrix elements of the hfs and electric dipole operators are found by means of the time-dependent HartreeFock (TDHF) method 28, 34]. This method is equivalent to the well-known random-phase approximation (RPA). In the TDHF method, single-electron wave functions are presented in the form $\psi=\psi_{0}+\delta \psi$, where $\psi_{0}$ is unperturbed wave function. It is an eigenstate of the RHF Hamiltonian $\hat{H}_{0}:\left(\hat{H}_{0}-\epsilon_{0}\right) \psi_{0}=0 . \quad \delta \psi$ is the correction due to external field. It can be found be solving the TDHF equation

$$
\left(\hat{H}_{0}-\epsilon_{0}\right) \delta \psi=-\delta \epsilon \psi_{0}-\hat{F} \psi_{0}-\delta \hat{V}^{N-1} \psi_{0},
$$

where $\delta \epsilon$ is the correction to the energy due to external field ( $\delta \epsilon \equiv 0$ for the electric dipole operator), $\hat{F}$ is the operator of the external field $\left(\hat{H}_{h f s}\right.$ or $\left.e \boldsymbol{E} \cdot \boldsymbol{r}\right)$, and $\delta \hat{V}^{N-1}$ is the correction to the self-consistent potential of the core due to external field. The TDHF equations are solved self-consistently for all states in the core. Then matrix elements between any (core or valence) states $n$ and $m$ are given by

$$
\left\langle\psi_{n}\left|\hat{F}+\delta \hat{V}^{N-1}\right| \psi_{m}\right\rangle
$$

The best results are achieved when $\psi_{n}$ and $\psi_{m}$ are Brueckner orbitals calculated with rescaled correlation potential $\hat{\Sigma}$.

We use equation (15) for all hfs and electric dipole matrix elements in (8), (9), and (10) except for the ground state hfs matrix element in (10) where we use experimental data.

To check the accuracy of the calculations we perform calculations of the hfs in the ground state and of the static scalar polarizabilities of the atoms. Polarizabilities are given by the expression

$$
\alpha_{0}(a)=\frac{2}{3} \sum_{m} \frac{|\langle a|| r|| m\rangle|^{2}}{\left(\epsilon_{a}-\epsilon_{m}\right)}
$$

which is very similar to the term (10) for the frequency shift. The most important difference is that the energy
TABLE II: Contributions to the hyperfine structure of the ground state of $\mathrm{Rb}, \mathrm{Cs}, \mathrm{Ba},{ }^{+}, \mathrm{Yb}^{+}$and $\mathrm{Hg}^{+}$(MHz); comparison with experiment.

\begin{tabular}{lrrrrrrr}
\hline \hline \multicolumn{1}{c}{ Atom } & Brueck & RPA & Str & Norm & Total & \multicolumn{1}{c}{ Exp } \\
\hline${ }^{87} \mathrm{Rb}$ & $5 s$ & 2888 & 559 & -27 & -33 & 3386 & $3417^{a}$ \\
${ }^{133} \mathrm{Cs}$ & $6 s$ & 1957 & 355 & -10 & -31 & 2278 & $2298^{b}$ \\
${ }^{137} \mathrm{Ba}^{+}$ & $6 s$ & 3509 & 601 & -21 & -73 & 4016 & $4019^{c}$ \\
${ }^{171} \mathrm{Yb}^{+}$ & $6 s$ & 11720 & 1540 & -248 & -247 & 12764 & $12645(2)^{d}$ \\
${ }^{199} \mathrm{Hg}^{+}$ & $6 s$ & 38490 & 3873 & 288 & -1483 & 41169 & $40507^{e}$ \\
\hline \hline
\end{tabular}

${ }^{a}$ Reference 35 .

${ }^{b}$ Reference [35].

${ }^{c}$ References 36] and 37].

${ }^{d}$ Reference 38|.

${ }^{e}$ Reference 39].

denominator is squared in term (10) but not in (16). This means better convergence with respect to the summation over complete set of states for term (10) than for (16). Therefore, if good accuracy is achieved for polarizabilities, even better accuracy should be expected for the term (10) (see also Ref. [1]]).

However, the behavior of the other two terms, (8) and (9), is very different and calculation of polarizabilities tells us little about accuracy for these terms. Therefore, we also perform detailed calculations of the hfs constants of the ground state. Inclusion of core polarization (second term in (15)) involves summation over the complete set of states similar to what is needed for term (8). Comparing experimental and theoretical hfs is a good test of the accuracy of this term.

In addition to term (15), we also include two smaller contributions to the hfs: structure radiation and the correction due to the change of the normalization of the wave function. The structure radiation term can be presented in the form

$$
A_{s t r}=\left\langle\psi_{n}|\delta \hat{\Sigma}| \psi_{n}\right\rangle
$$

where $\delta \hat{\Sigma}$ is the correction to the correlation potential due to external hfs field. The normalization term is

$$
A_{\text {norm }}=-A_{n}\left\langle\psi_{n}\left|\frac{\partial \hat{\Sigma}}{\partial \epsilon}\right| \psi_{n}\right\rangle,
$$

where $A_{n}$ is given by (15) with $m=n$.

The results for hfs are presented in Table II Here column marked as 'Brueck' corresponds to the $\left\langle\psi_{n}|\hat{F}| \psi_{n}\right\rangle$ matrix element. The column marked as RPA is the core polarization correction given by $\left\langle\psi_{n}\left|\delta \hat{V}^{N-1}\right| \psi_{n}\right\rangle$, the notation 'Str' stands for structure radiation given by (17), and 'Norm' is the renormalization contribution given by (18). In all cases $\psi_{n}$ is the Bruckner orbital corresponding to the ground state of the atom or ion, calculated with the rescaled correlation potential $\hat{\Sigma}$. All-order $\hat{\Sigma}^{(\infty)}$ is used for $\mathrm{Rb}, \mathrm{Cs}$ and $\mathrm{Ba}^{+}$. Second-order $\hat{\Sigma}^{(2)}$ is used for $\mathrm{Yb}^{+}$and $\mathrm{Hg}^{+}$. Comparing the final theoretical results with experiment shows that the theoretical accuracy is 
TABLE III: Static polarizabilities $\alpha_{0}$ of $\mathrm{Rb}, \mathrm{Cs}, \mathrm{Ba},{ }^{+}, \mathrm{Yb}^{+}$ and $\mathrm{Hg}^{+}$in different approximations $\left(a_{0}^{3}\right)$.

\begin{tabular}{|c|c|c|c|c|c|c|}
\hline \multicolumn{2}{|c|}{ Atom } & $\hat{\Sigma}$ & $\alpha_{v}^{a}$ & $\alpha_{c}^{b}$ & Total & Other works \\
\hline \multirow[t]{4}{*}{${ }^{87} \mathrm{Rb}$} & \multirow[t]{4}{*}{$5 s$} & $\hat{\Sigma}^{(2) c}$ & 292.7 & 9.1 & 301.8 & $329(23)^{f}$ \\
\hline & & $\lambda \hat{\Sigma}^{(2) d}$ & 309.7 & 9.1 & 318.8 & $293(6)^{g}$ \\
\hline & & $\hat{\Sigma}^{(\infty) e}$ & 312.4 & 9.1 & 321.5 & $318.6(6)^{h}$ \\
\hline & & $\lambda \hat{\Sigma}^{(\infty) d}$ & 310.5 & 9.2 & 319.7 & $318.5(6)^{i}$ \\
\hline \multirow[t]{4}{*}{${ }^{133} \mathrm{Cs}$} & \multirow[t]{4}{*}{$6 s$} & $\hat{\Sigma}^{(2) c}$ & 343.8 & 15.3 & 359.1 & $401.0(6)^{j}$ \\
\hline & & $\lambda \hat{\Sigma}^{(2) d}$ & 383.5 & 15.4 & 399.0 & $401.5^{h}$ \\
\hline & & $\hat{\Sigma}^{(\infty) e}$ & 384.0 & 15.5 & 399.5 & $400.4^{k}$ \\
\hline & & $\lambda \hat{\Sigma}^{(\infty) d}$ & 384.4 & 15.5 & 399.9 & $400.6(1.0)^{l}$ \\
\hline \multirow[t]{4}{*}{${ }^{137} \mathrm{Ba}^{+}$} & \multirow[t]{4}{*}{$6 s$} & $\hat{\Sigma}^{(2) c}$ & 104.1 & 9.8 & 113.8 & \\
\hline & & $\lambda \hat{\Sigma}^{(2) d}$ & 112.5 & 9.9 & 122.4 & \\
\hline & & $\hat{\Sigma}^{(\infty) e}$ & 112.8 & 9.9 & 122.7 & \\
\hline & & $\lambda \hat{\Sigma}^{(\infty) d}$ & 112.7 & 9.9 & 122.7 & \\
\hline \multirow[t]{2}{*}{${ }^{171} \mathrm{Yb}^{+}$} & \multirow[t]{2}{*}{$6 s$} & $\hat{\Sigma}^{(2) c}$ & 50.9 & 6.2 & 57.1 & \\
\hline & & $\lambda \hat{\Sigma}^{(2) d}$ & 55.4 & 6.1 & 61.5 & \\
\hline \multirow[t]{2}{*}{${ }^{199} \mathrm{Hg}^{+}$} & $6 s$ & $\hat{\Sigma}^{(2) c}$ & 10.5 & 7.7 & 18.2 & \\
\hline & & $\lambda \hat{\Sigma}^{(2) d}$ & 11.4 & 7.6 & 19.0 & \\
\hline
\end{tabular}

${ }^{a}$ Polarizability due to valence electron.

${ }^{b}$ Polarizability of the core.

${ }^{c} \hat{\Sigma}^{(2)}$ is the second-order correlation potential.

${ }^{d}$ Rescaled $\hat{\Sigma}$. See Table $\prod$ for the values of rescaling factors $\lambda$.

${ }^{e} \hat{\Sigma}^{(\infty)}$ is the all-order correlation potential.

$f_{\text {Measurements, Ref. } 20 \text {. }}$.

${ }^{g}$ Measurements, Ref. 21].

${ }^{h}$ Calculations, Ref. 22.

${ }^{i}$ Calculations, Ref. [23].

${ }^{j}$ Measurements, Ref. 24].

${ }^{k}$ Calculations, Ref. 25].

${ }^{l}$ Calculations, Ref. 11].

within $1 \%$ for all atoms except $\mathrm{Hg}^{+}$where it is $1.6 \%$. If the structure radiation and normalization are neglected, accuracy for $\mathrm{Rb}$ and $\mathrm{Cs}$ remains within $1 \%$, accuracy for $\mathrm{Ba}^{+}$becomes about $2 \%$ and accuracy for $\mathrm{Yb}^{+}$and $\mathrm{Hg}^{+}$ becomes close to $5 \%$.

The results for polarizabilities, calculated in different approximations, are presented in Table III Pure $a b$ initio results obtained with $\hat{\Sigma}^{(\infty)}$ and results obtained with rescaled correlation potential operators $\hat{\Sigma}^{(2)}$ and $\hat{\Sigma}^{(\infty)}$ are very close to each other and to other calculations and measurements.

\section{RESULTS AND DISCUSSION}

Table IV presents contributions of terms (8), (9) and (10) into the total frequency shift of the hfs transitions for the ground states of ${ }^{87} \mathrm{Rb},{ }^{133} \mathrm{Cs},{ }^{137} \mathrm{Ba}^{+},{ }^{171} \mathrm{Yb}^{+}$ and ${ }^{199} \mathrm{Hg}^{+}$calculated in different approximations. Term (10) dominates in all cases, while term (9) is small but still important at least for $\mathrm{Rb}, \mathrm{Cs}$ and $\mathrm{Ba}^{+}$. Results obtained with $\hat{\Sigma}^{(2)}$ and $\hat{\Sigma}^{(\infty)}$ differ significantly (up to $14 \%$ for Cs). However, after rescaling the results for both $\hat{\Sigma}^{(2)}$
TABLE IV: Contribution of terms (8), (9), and (10) to the frequencies of the hyperfine transitions in the ground state of $\mathrm{Rb}, \mathrm{Cs}, \mathrm{Ba},{ }^{+}, \mathrm{Yb}^{+}$and $\mathrm{Hg}^{+}\left(\delta \nu_{0} / E^{2} \times 10^{-10} \mathrm{~Hz} /(\mathrm{V} / \mathrm{m})^{2}\right)$ in different approximations.

\begin{tabular}{|c|c|c|c|c|c|c|}
\hline \multicolumn{2}{|c|}{ Atom } & $\hat{\Sigma}$ & (8) & (9) & (10) & Total \\
\hline \multirow[t]{4}{*}{${ }^{87} \mathrm{Rb}$} & $5 s$ & $\hat{\Sigma}^{(2) a}$ & -0.5457 & 0.0147 & -0.6692 & -1.2003 \\
\hline & & $\lambda \hat{\Sigma}^{(2) b}$ & -0.5668 & 0.0154 & -0.6894 & -1.2409 \\
\hline & & $\hat{\Sigma}^{(\infty) c}$ & -0.5640 & 0.0156 & -0.7034 & -1.2518 \\
\hline & & $\lambda \hat{\Sigma}^{(\infty) b}$ & -0.5620 & 0.0154 & -0.6972 & -1.2437 \\
\hline \multirow[t]{4}{*}{${ }^{133} \mathrm{Cs}$} & $6 s$ & $\hat{\Sigma}^{(2) a}$ & -0.9419 & 0.0210 & -1.0722 & -1.9931 \\
\hline & & $\lambda \hat{\Sigma}^{(2) b}$ & -1.0239 & 0.0229 & -1.2688 & -2.2697 \\
\hline & & $\hat{\Sigma}^{(\infty) c}$ & -1.0148 & 0.0232 & -1.2706 & -2.2622 \\
\hline & & $\lambda \hat{\Sigma}^{(\infty) b}$ & -1.0167 & 0.0230 & -1.2695 & -2.2632 \\
\hline \multirow[t]{4}{*}{${ }^{137} \mathrm{Ba}^{+}$} & $6 s$ & $\hat{\Sigma}^{(2) a}$ & -0.1027 & 0.0034 & -0.1568 & -0.2561 \\
\hline & & $\lambda \hat{\Sigma}^{(2) b}$ & -0.1095 & 0.0036 & -0.1768 & -0.2827 \\
\hline & & $\hat{\Sigma}^{(\infty) c}$ & -0.1104 & 0.0037 & -0.1778 & -0.2845 \\
\hline & & $\lambda \hat{\Sigma}^{(\infty) b}$ & -0.1104 & 0.0037 & -0.1773 & -0.2841 \\
\hline \multirow[t]{2}{*}{${ }^{171} \mathrm{Yb}^{+}$} & $6 s$ & $\hat{\Sigma}^{(2) a}$ & -0.0672 & 0.0009 & -0.0866 & -0.1529 \\
\hline & & $\lambda \hat{\Sigma}^{(2) b}$ & -0.0714 & 0.0011 & -0.1003 & -0.1706 \\
\hline \multirow[t]{2}{*}{${ }^{199} \mathrm{Hg}^{+}$} & $6 s$ & $\hat{\Sigma}^{(2) a}$ & -0.0242 & 0.0000 & -0.0296 & -0.0538 \\
\hline & & $\lambda \hat{\Sigma}^{(2) b}$ & -0.0263 & 0.0000 & -0.0335 & -0.0598 \\
\hline
\end{tabular}

${ }^{a} \hat{\Sigma}^{(2)}$ is the second-order correlation potential.

${ }^{b}$ Rescaled $\hat{\Sigma}$. See Table $\prod$ for the values of rescaling factors $\lambda$.

${ }^{c} \hat{\Sigma}(\infty)$ is the all-order correlation potential.

and $\hat{\Sigma}^{(\infty)}$ come within a fraction of a per cent of each other. Naturally, rescaling has a larger effect on results obtained with $\hat{\Sigma}^{(2)}$. This means that the rescaling really imitates the effect of higher-order correlations and should lead to more accurate results. Comparing the results obtained with $\hat{\Sigma}^{(\infty)}$, rescaled $\hat{\Sigma}^{(\infty)}$ and rescaled $\hat{\Sigma}^{(2)}$ gives a reasonable estimation of the accuracy of calculations. If we also combine this with the calculation of the hfs discussed above we can safely assume that the accuracy of the calculations for $\mathrm{Rb}, \mathrm{Cs}$ and $\mathrm{Ba}^{+}$is on the level of $1 \%$. Note that the frequency shift due to black body radiation can be a little larger $(\sim 1 \%)$ due to the effect of frequency distribution at finite temperature.

For $\mathrm{Yb}^{+}$and $\mathrm{Hg}^{+}$we only have results with rescaled $\hat{\Sigma}^{(2)}$ and not rescaled $\hat{\Sigma}^{(\infty)}$. They differ by about $11 \%$. However, there are strong reasons to believe that the results obtained with rescaling are more accurate. This is supported by calculations for $\mathrm{Rb}, \mathrm{Cs}$ and $\mathrm{Ba}^{+}$as well as our experience with rescaling used in many earlier works. Therefore, the calculation of the hfs discussed above gives a more realistic estimation of the accuracy for $\mathrm{Yb}^{+}$and $\mathrm{Hg}^{+}$which is about $5 \%$.

Table IV presents values of $k$ (see formula (4)). To obtain the frequency shift at finite temperature one needs to convert $k$ into $\beta$ using formula (5) and substitute $\beta$ into equation (3). For accurate results one also needs to know the values of $\epsilon$. We calculate them using formula (13) in a very similar way as we calculate parameters $k$. Our final values of $k, \beta$ and $\epsilon$ are presented in Table $\mathbb{\nabla}$ Parameter 
TABLE V: Final results for the parameters $k$ $\left(10^{-10} \mathrm{~Hz} /(\mathrm{V} / \mathrm{m})^{2}\right), \quad \beta\left(10^{-14}\right)$ and $\epsilon$ of the black-body radiation frequency shift for $\mathrm{Rb}, \mathrm{Cs}, \mathrm{Ba},{ }^{+}, \mathrm{Yb}^{+}$and $\mathrm{Hg}^{+}$.

\begin{tabular}{lllll}
\hline \hline \multicolumn{1}{c}{ Atom } & & \multicolumn{1}{c}{$k$} & \multicolumn{1}{c}{$\beta$} & $\epsilon$ \\
\hline${ }^{87} \mathrm{Rb}$ & $5 s$ & $-1.24(1)$ & $-1.26(1)$ & 0.011 \\
${ }^{133} \mathrm{Cs}$ & $6 s$ & $-2.26(2)$ & $-1.70(2)$ & 0.013 \\
${ }^{137} \mathrm{Ba}^{+}$ & $6 s$ & $-0.284(3)$ & $-0.245(2)$ & 0.004 \\
${ }^{171} \mathrm{Yb}^{+}$ & $6 s$ & $-0.171(9)$ & $-0.094(5)$ & 0.002 \\
${ }^{199} \mathrm{Hg}^{+}$ & $6 s$ & $-0.060(3)$ & $-0.0102(5)$ & 0.0005 \\
\hline \hline
\end{tabular}

$\epsilon$ for Cs was estimated in single-resonance approximation in Ref [1] and found to be 0.014. This value is in good agreement with our accurate calculations.

The frequency shifts of some alkali metal atoms have been calculated and measured previously. We present previous results for the atoms and ions for which we perform calculations in Table VI together with our final results.

There is some disagreement for cesium. Our result is in good agreement with early measurements [2, 3, 5] and $a b$ initio calculations [9, 10] while recent measurements [6, 7] and semiempirical calculations $[8,11,12$ give the value which is about $10 \%$ smaller. Less accurate measurements of Bauch and Schröder [4] cover both cases. We cannot comment on disagreement between experimental results. However, the source of disagreement between theoretical results seems to be clear. It comes from the contribution of the continuum spectrum to the summation over the complete set of states in term (8). This term has offdiagonal hfs matrix elements between the ground state and excited states. Since the hfs interaction is localized over short distances $\left(\sim a_{0} / Z\right)$ it emphasizes the contribution of states with high energies including states in the continuum (since $\Delta p \Delta x \geq \hbar$, a small area of localization $(\Delta x)$ allows high momentum $(p)$ and thus high energy). In our calculations the contribution of states above $7 p$ into term (8) is $-0.35 \times 10^{-1} \mathrm{~Hz} /(\mathrm{V} / \mathrm{m})^{2}$ which is $15 \%$ of the total answer.

In contrast, states above $7 p$ contribute only about $0.05 \%$ of the total value of term (10). This is because the summation goes over the matrix elements of the electric dipole operator which is large on large distances and thus suppresses the contribution of high-energy states. It is not surprising therefore that a semiempirical consideration, involving only discrete spectrum states, gives very good results for the atomic polarizabilities (see, e.g. 11]). However, let us stress once more that the calculation of polarizabilities checks only term (10) and tells us very little about the accuracy of other two terms, (8) and (9).

The contribution of the states above $7 p$ is even more important for term (9). Their contribution is about 30\% of the total value of this term. However, the term itself is small and its accurate treatment is less important.

In ab initio calculations by Lee et al [9] summation over complete set of states is reduced to solving of a radial
TABLE VI: Electrostatic frequency shifts for the hyperfine transitions of $\mathrm{Rb}, \mathrm{Cs}, \mathrm{Ba}^{+}, \mathrm{Yb}^{+}$and $\mathrm{Hg}^{+}\left(\delta \nu_{0} / E^{2} \times 10^{-10}\right.$ $\left.\mathrm{Hz} /(\mathrm{V} / \mathrm{m})^{2}\right)$; comparison with other calculations and measurements.

\begin{tabular}{cccllll}
\hline \hline \multicolumn{1}{c}{ Atom } & $\begin{array}{r}\text { This } \\
\text { work }\end{array}$ & \multicolumn{1}{c}{$\begin{array}{c}\text { Other } \\
\text { calculations }\end{array}$} & Ref & Measurements & Ref \\
& & & \\
\hline${ }^{87} \mathrm{Rb}$ & $5 s$ & $-1.24(1)$ & -1.2094 & {$[9]$} & $-1.23(3)$ & {$[3]$} \\
${ }^{133} \mathrm{Cs}$ & $6 s$ & $-2.26(2)$ & $-1.9(2)$ & {$[8]$} & $-2.29(7)$ & {$[2]$} \\
& & & -2.2302 & {$[9]$} & $-2.25(5)$ & {$[3]$} \\
& & -2.28 & {$[10]$} & $-2.17(26)$ & {$[4]$} \\
& & $-1.97(9)$ & {$[11]$} & $-2.271(4)$ & {$[5]$} \\
& & $-2.06(1)$ & {$[12]$} & $-1.89(12)$ & {$[6]$} \\
${ }^{137} \mathrm{Ba}^{+}$ & $6 s$ & $-0.284(3)$ & -0.27 & {$[1]$} & & \\
${ }^{171} \mathrm{Yb}^{+}$ & $6 s$ & $-0.171(9)$ & & & \\
${ }^{199} \mathrm{Hg}^{+}$ & $6 s$ & $-0.060(3)$ & -0.058 & {$[1]$} & \\
\hline \hline
\end{tabular}

equation (equations of this type are often called Sternheimer equation after one of the authors of this work). This approach does include the contribution of the continuum spectrum and the result is in very good agreement with ours (see Table VI).

In other ab initio calculations by Pal'chikov et al 10] summation is done via Green functions. This corresponds to summation over the complete set of states and does include the continuum spectrum. Again, the result is in very good agreement with other $a b$ initio calculations (9] and the present work).

Recent calculations by Beloy et al 13 applied a mixed approach, with extensive use of experimental data for lower cesium states and ab initio summation over higher states including continuum. The result is in good agreement with fully $a b$ initio calculations.

In contrast, analysis performed in 8, 11, 12 is limited to discrete spectrum. Adding $-0.34 \times 10^{-1} \mathrm{~Hz} /(\mathrm{V} / \mathrm{m})^{2}$ (which is total tail contribution from all three terms (8), (9) and (10) found in our calculation) to the results of Feitchner et al [8] and Micalizio et al [1] brings them to excellent agreement with $a b$ initio calculations. The same modification of the result by Ulzega et al 12 makes it a little bit too large but still closer to other results than without the tail contribution.

\section{CONCLUSION}

We have performed calculations of the frequency shift of the ground state hyperfine transition for several atoms and ions caused by a static electric field which can be used to evaluate the effect of blackbody radiation on the frequency of the microwave atomic clock transitions. The size of this shift is comparable to the current error in the measurements of the energy shift caused by variation of $\alpha$ and so needs to be taken into account in laboratory measurements placing limits upon $\alpha$ variation. 
Detailed analysis of the calculations for cesium reveal the source of disagreement between different theoretical approaches. This seems to be contribution of the continuum spectrum into summation over complete set of states which was neglected in semiempirical calculations. Restoring the tail contribution in works where it was neglected brings all theoretical results in good agreement with each other.

\section{Acknowledgments}

This work was suggested by Bruce Warrington. We are grateful for his initial suggestion and useful discussions. We are also grateful to S. Ulzega, W. Itano and A. Derevianko for useful comments and references. The work was supported by the Australian Research Council.
[1] W. M. Itano, L. L. Lewis, and D. J. Wineland, Phys. Rev. A 25, 1233 (1982).

[2] R. D. Haun and J. R. Zacharias, Phys. Rev. 107, 107 (1957).

[3] J. R. Mowat, Phys. Rev. A 5, 1059 (1972).

[4] A. Bauch and R. Schröder, Phys. Rev. Lett. 78, 622 (1997).

[5] E. Simon, P. Laurent, and A. Clairon, Phys. Rev. A 57, 436 (1998).

[6] F. Levi, D. Calonico, L. Lorini, S. Micalizio, and A. Godone, Phys. Rev. A, 70, 033412 (2004).

[7] A. Godone, D. Calonico, F. Levi, S. Micalizio, and Claudio Calosso, Phys. Rev. A, 71, 063401 (2005).

[8] J. D. Feitchner, M. E. Hoover and M. Mitzushima, Phys. Rev. 137, A702 (1965).

[9] T. Lee, T. P. Das, and R. M. Sternheimer, Phys. Rev. A 11, 1784 (1975).

[10] V. G. Pal'chikov, Yu. S. Domnin, and A. V. Novoselov, J. Opt. B: Quantum Semiclassical Opt., 5, S131 (2003).

[11] S. Micalizio, A. Godone, D. Calonico, F. Levi, and L. Lorini, Phys. Rev. A, 69, 053401 (2004).

[12] S. Ulzega, A. Hofer, P. Moroshkin, and A. Weis, e-print: physics/0604233

[13] K. Beloy, U. I. Safronova, and A. Derevianko, e-print: physics/0606048 accepted to Phys. Rev. Lett.

[14] E. Peik, B. Lipphardt, H. Schnatz, T. Schneider, Chr. Tamm, and S. G. Karshenboim, Phys. Rev. Lett. 93, 170801 (2004).

[15] S. G. Karshenboim, V. V. Flambaum, and E. Peik, in G.W.F. Drake, Handbook on Atomic, Molecular and $O p$ tical Physics (Springer, 2005), p. 455; physics/0410074

[16] M. Fischer et al., Phys. Rev. Lett. 92, 230802 (2004).

[17] S. Bize, S. A. Diddams, U. Tanaka, C. E. Tanner, W. H. Oskay, R. E. Drullinger, T. E. Parker, T. P. Heavner, S. R. Jefferts, L. Hollberg, W. M. Itano, and J. C. Bergquist, Phys. Rev. Lett. 90, 150802 (2003).

[18] B. Warrington, private communication.

[19] E. J. Angstmann, V. A. Dzuba, and V. V. Flambaum, e-print: physics/0606249 accepted to Phys. Rev. Lett.

[20] R. W. Molof, H. L. Schwartz, T. M. Miller, and B. Bederson, Phys. Rev. A, 10, 1131 (1974).
[21] W. D. Hall and J. C. Zorn, Phys. Rev. A, 10, 1141 (1974).

[22] A. Derevianko, W. R. Johnson, M. S. Safronova, J. F. Babb, Phys. Rev. Lett., 82, 3589 (1999).

[23] M. S. Safronova, C. J. Williams, and C. W. Clark, Phys. Rev. A, 67, 040303(R) (2003).

[24] J. M. Amini and H. Gould, Phys. Rev. Lett., 91, 153001 (2003).

[25] A. Derevianko and S. G. Porsev, Phys. Rev. A, 65, 53403 (2002).

[26] L. D. Landau and E. M. Lifshitz, Quantum Mechanics: Non-relativistic Theory, (Pergamon, 1977).

[27] V. A. Dzuba and V. V. Flambaum, Phys. Rev. A 62, 052101 (2000).

[28] V. A. Dzuba, V. V. Flambaum, P. G. Silvestrov, and O. P. Sushkov, J. Phys. B: At. Mol. Phys. 20, 1399 (1987).

[29] V. A. Dzuba, V. V. Flambaum, and O. P. Sushkov, Phys. Lett. A 141, 147 (1989).

[30] W. R. Johnson, and J. Sapirstein, Phys. Rev. Lett. 57, 1126 (1986).

[31] W. R. Johnson, S. A. Blundell, and J. Sapirstein, Phys. Rev. A 37, 307 (1988).

[32] V. A. Dzuba, V. V. Flambaum, and O. P. Sushkov, Phys. Rev. A 51, 3454 (1995).

[33] V. A. Dzuba, V. V. Flambaum, and J. S. M. Ginges, Phys. Rev. A 63, 062101 (2001).

[34] V. A. Dzuba, V. V. Flambaum, and O. P. Sushkov, J. Phys. B: At. Mol. Phys. 17, 1953 (1984).

[35] G. H. Fuller, and V. W. Cohen, Nucl. Data, Sect. A, 5, 433 (1969).

[36] J. Vetter, M. Stuke, and E. W. Weber, The Euro. Phys. J. A 273, 129 (1975).

[37] S. Trapp, G. Marx, G. Tommaseo, A. Klaas, A. Drakoudis, G. Revalde, G. Szawiola, G. Werth, Hyperfine Interactions, 127, 57 (2000).

[38] A.-M. Mårtensson-Pendrill, D. S. Gough, and P. Hannaford, Phys. Rev. A, 49, 3351 (1994).

[39] D. J. Berkeland, J. D. Miller, B. C. Young, J. C. Bergquist, W. M. Itano, and D. J. Wineland, Proc. SPIE 3270, 138 (1998). 